\title{
Rapid Method of Small RNA Purification in Applications Involving RNA-Protein Interactions
}

\author{
Marimuthu Citartan ${ }^{1}$, Soo-Choon Tan ${ }^{2}$, Thean-Hock Tang ${ }^{* 1}$ \\ ${ }^{1}$ Infectious Disease Cluster, Advanced Medical \& Dental Institute (AMDI), University Sains Malaysia, Penang, Malaysia \\ ${ }^{2}$ Institute for Research in Molecular Medicine (INFORMM), Universiti Sains Malaysia, Penang, Malaysia \\ tangth@amdi.usm.edu.my
}

\begin{abstract}
In vitro analysis of RNA-protein interaction requires good quality RNA. Most of the current methods of RNA purification are tedious, time consuming and involves incomplete separation of degraded and fulllength RNA. Moreover, these methods are also associated with hazardous materials such as ethidium bromide and phenol: chloroform. In addition, impurities co-purified with RNA samples can be potential contaminants that can compromise output signal such as signal measurement in Surface Plasmon Resonance (SPR). Previously, we have reported on the fast and costeffective method of small RNA purification from polyacrylamide gel (PAGE) as an alternative in alleviating problems often experienced with the current methods of purification. Optimal temperaturedependant elution of the small RNA was within the range of 50-60 ${ }^{\circ} \mathrm{C}$. Our method of small RNA purification can be employed towards preparing RNA for RNA-protein interaction assays, since most of these analyses involve RNA with the size of 20-140 nucleotides long as the ligand. In this review, we attempt to overview the potential applicability of our small RNA purification method in several assays of RNA-protein interaction.
\end{abstract}

Keywords- RNA Purification; RNA-Protein Interaction

\section{INTRODUCTION}

Since the discovery that RNA acts both as information carrier and regulatory molecules, RNA has amassed great interest in a plethora of studies such as RNomics (Chinni et al., 2010; Tang et al., 2002a, Tang et al., 2002b), real-time PCR (Armstrong et al., 2012) and RNA-protein interaction (Gopinath et al., 2008). It is construed that RNA works together with protein in performing various functions in vivo. In prokaryotes, 23S, 5.8S and 5S rRNA (ribosomal RNA) form complex with ribosomal protein to form $50 \mathrm{~S}$ ribosomal subunit, while the association of 16S rRNA with ribosomal protein gives rise to $30 \mathrm{~S}$ ribosomal subunit. Both of these ribosomal subunits are integral components of ribosome that translates mRNA into protein. In eukaryotes, guide strand of the siRNA (small interfering RNA) integrates with the
Argonaute protein of the RNA induced silencing complex (RISC). This complex is then guided to the complementary target RNA to initiate gene silencing. In addition, U-rich (snRNA) small nuclear RNAs integrate with the proteins to form snRNP (small ribonucleoprotein complex), which is involved in the splicing of pre-mRNA in eukaryotes. On the other hand, recent studies demonstrated the folding of small RNA hairpin (8-nucleotide long) that consisted of a tetraloop and a two-basepair stem (Zuo et al., 2010). These conformations enable small RNA (or of equivalent length) to embed inside the clefts or binding pockets on the surface of protein. Thus, these in vivo RNA-protein complex formations and the ability of the small RNAs to form a wide variety of conformations prompt scientists to analyze and manipulate the interaction of RNA with protein in vitro (Leontis et al., 2006). In order to analyze RNA-protein interaction, the purity and quality of the RNA is of utmost importance and the purification should be carried out in the shortest amount of time possible.

In the previous paper, we reported on the rapid method of purifying small RNA with minimal cost while maintaining the yield and purity. Following visualization of the RNA run on the urea-denaturing PAGE under short wavelength $(254 \mathrm{~nm})$ u.v, the band is selectively excised. Elution of the small RNA was carried out at 30, 40, 50, 60 and $80^{\circ} \mathrm{C}$ for 30 minutes in water. Subsequently, these small RNA fragments were recovered by ethanol precipitation. Elution at $50-60^{\circ} \mathrm{C}$ for 30 minutes is the optimum temperature to recover small RNA. The advantage of this method is that it does not require hazardous materials such as ethidium bromide and phenol-chloroform. Time spent on the small RNA purification is also reduced without compromising quality (Citartan et al., 2012). In this review, several possible applications (Systematic Evolution of Ligands by Exponential Enrichment (SELEX), filter-binding and gel shift assay as well as RNA-protein mapping analyses) of the RNA purified using this method will be discussed. In addition, label-free applications of the RNAprotein analysis such as Surface Plasmon Resonance, waveguide based sensor, UV-vis-NIR spectroscopy based measurements are also overviewed (Figure 1). 

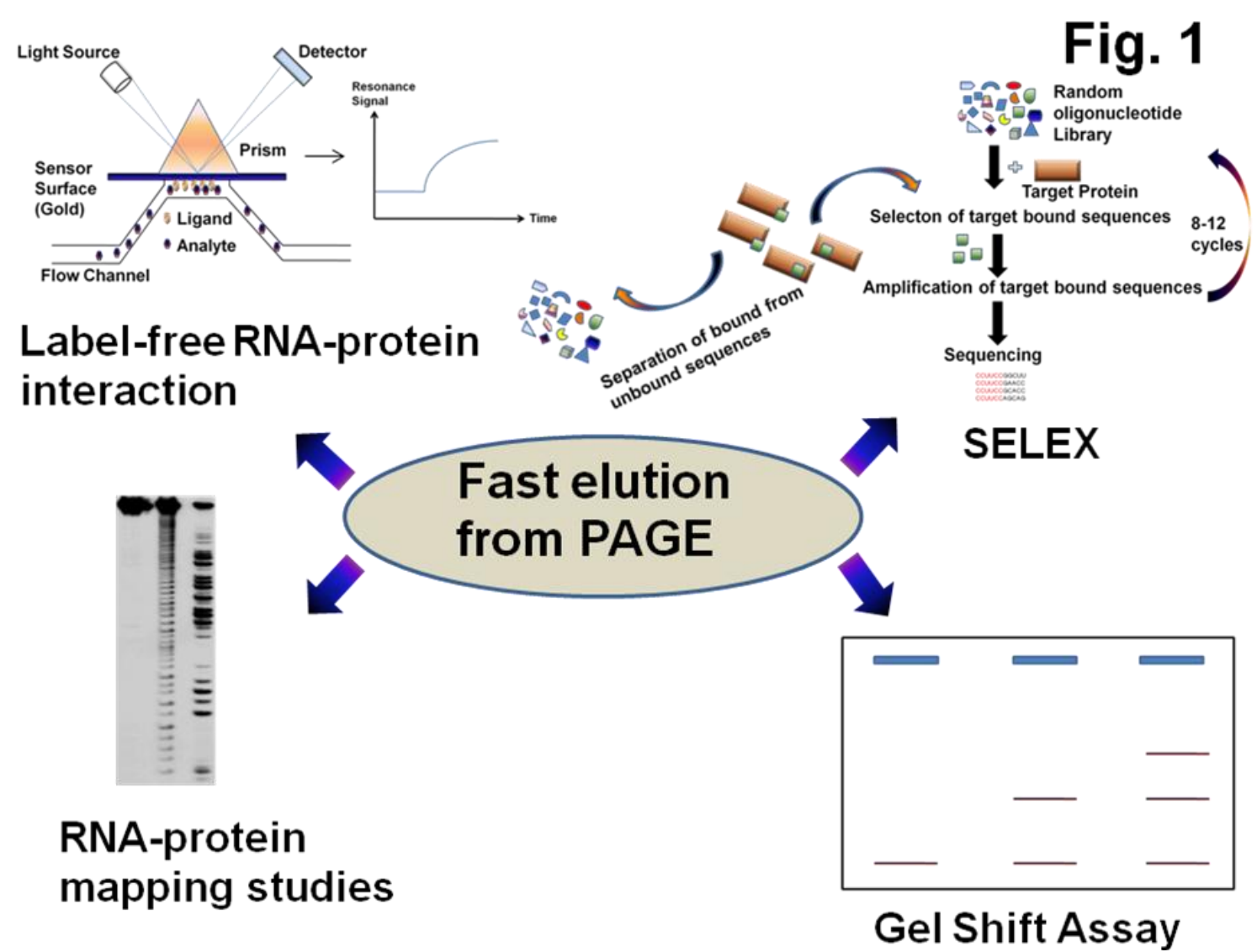

Figure 1 Several applications of the fast elution method for RNA purification

\section{FILTER-BINDING AND GEL SHIFT ASSAY}

Filter-Binding assay is one of the methods to study RNA-protein interactions. In this assay, RNA-protein complex is retained on the surface of nitrocellulose filter membrane, while the unbound RNA passes through the filter. The amount of the labeled RNA-protein complex retained on the surface of the filter is quantified by scintillation counting, a method for measuring radiation from beta-emitting nuclides (Stockley et al., 2009). Following this, dissociation constant is calculated, as equal to the concentration of protein at which $50 \%$ of the radiolabeled RNA is bound to protein (Figure 2a). In the SELEX experiment involving hemagglutinin of human influenza virus B, Gopinath et al. (2006) had performed filter-binding assay on one of the radiolabeled clones from cycle 9 of SELEX and calculated the dissociation constant to be at $44 \pm 6 \mathrm{nM}$. Thus, labeling of RNA with radioactive tags represents a crucial step in the filter-binding assay. The RNA for filter-binding assay can be radioactively labeled at 5 '-end or 3 '-end by [gamma- ${ }^{32} \mathrm{P}$ ] ATP or [5'-32P] pCp (cytidine-3', 5'-bis-phosphate), respectively. However, this labeled RNA must be purified from the unincorporated radiolabeled nucleotides and labeling enzymes such as Polynucleotide Kinase and T4 RNA Ligase. The usage of spin column chromatography based on certain cutoff values can only remove these unincorporated nucleotides. However, the labeled RNA and the excess unlabeled RNA are still retained in the column. In addition, degraded RNA that is poorly labeled at 5' or 3'ends is also co-purified with the full-length labeled RNA. In order to obviate the problems associated with application of spin column, our small RNA purification protocol can be an alternative procedure for purification of radiolabeled RNA. In this procedure, the labeled RNA reaction mixture is first run on certain percentage of urea-denaturing PAGE based on the size of the RNA fragment. Following electrophoresis, the gel was exposed to X-ray film for a few minutes. After image development that identifies the precise position of the labeled RNA, the X-ray film is placed back onto the gel to trace the position and was marked on the gel (Figure $2 b$ ). This band was then cut, crushed and eluted at $55^{\circ} \mathrm{C}$ for 30 minutes in water followed by purification with the aid of ethanol and Dr. Gentle Precipitation Carrier. Dr. Gentle precipitation carrier allows recovery of low amount of nucleic acid (up to $5 \mathrm{ng} / \mathrm{uL}$ ) in the solution by centrifugation right immediately after incubation at room temperature. Analogous to Dr. Gentle, glycogen also acts as inert carrier of nucleic acid, precipitating nucleic acid after incubation at $-80^{\circ} \mathrm{C}$ for at least 15 minutes.

Similar method of purification can also be applied in purifying RNA transcripts obtained following hot in vitro transcription carried out with $[\alpha-32 \mathrm{P}]$ UTP or $[\alpha-32 \mathrm{P}]$ ATP. However, rather than exposing the PAGE gel containing the body-labeled RNA to X-ray film as explained previously, the labeled RNA can be visualized directly under short U.V wavelength as a clear discrete band (Figure 2c). This fast method of purification can minimize exposure to radiation and yields RNA of good quality. Our radiolabeled RNA purification method can also be harnessed in another assay 
of RNA-protein interaction analysis, which is the gel shift assay. This method also depends on the migration rate of the RNA fragment through the matrix of the non-denaturing PAGE. Migration rate is influenced by the three dimensional structure and the molecular weight of the RNA fragment. Thus, RNA-protein complex which has increased molecular weight migrates slower than the RNA fragment alone, providing information on the binding of the particular RNA with the protein (Ryder et al., 2008). Gel shift assay is widely used in SELEX experiments, as this assay can give answer as to whether the generated aptamer binds to the corresponding target protein. In this case, the assay is performed in single or dual concentration of protein such as in the RNA aptamer generation against Tat protein (Yamamoto et al., 2000). However, to calculate the dissociation constant of the aptamer against its target, incubation of the labeled RNA is carried out with different amounts of the target protein. Gopinath et al. (2008) have conducted gel shift assay of HutP-RNA complex. Various amounts of HutP proteins varying from 50-800 $\mathrm{nM}$ were incubated with labeled 21-mer RNA in binding buffer (15 mM HEPES pH 7.5, $30 \mathrm{mM} \mathrm{NaCl}, 10 \mathrm{mM}$ L-his, $10 \mathrm{mM}$ $\mathrm{MgCl}_{2}$ ) at room temperature. The result revealed a 1:1 ratio of RNA to protein complex formation. In both gel shift and filter-binding assay, the final concentration of the radiolabeled probe should be far less than the dissociation constant of the RNA-protein complex. This is because the low amount of the radiolabeled RNA ensures that the pool of free protein is not depleted. If the amount of probe is very high, dissociation constant cannot be calculated due to failure of achieving $50 \%$ of the protein bound labeled RNA, as all the radiolabeled RNA is bound to the protein. Thus, there is no need to start with high amount of RNA for labeling (as much as $1 \mu \mathrm{g}$ ). With the addition of suitable nucleic acid carrier, purified radiolabeled RNA is sufficient for dissociation constant calculation.

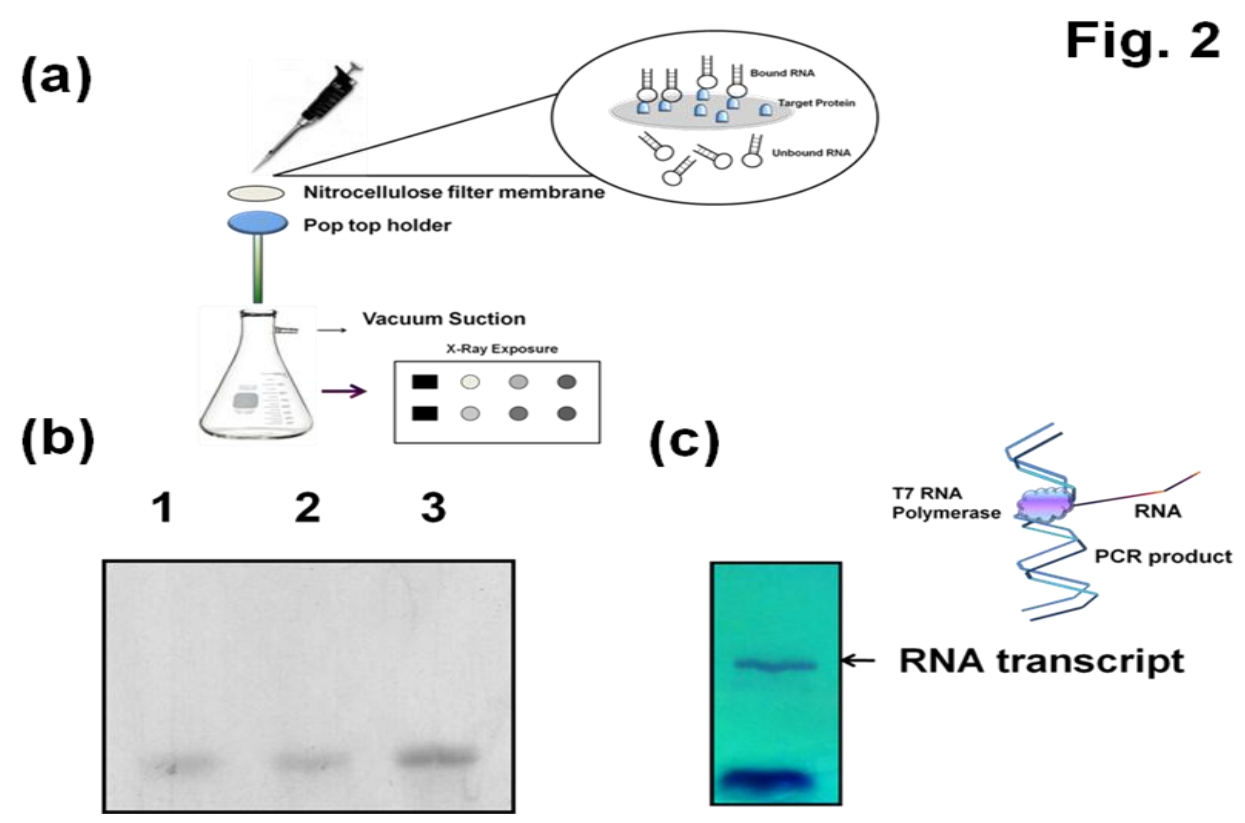

Figure 2 (a) Schematic representation of the filter-binding assay

Radiolabeled RNA is incubated with different concentrations of protein. These mixtures were filtered through the nitrocellulose filter membrane under vacuum suction, followed by washing step to remove non-specific binders. The filters were exposed to X-ray film and the intensity of the membrane which reflects amount of radioactivity retained was used to calculate dissociation constant

(b) One minute exposure of X-ray film on several radiolabeled RNA from cycle 12 of SELEX run on 12\% urea-denaturing PAGE

(c) Short wavelength $(254 \mathrm{~nm}) \mathrm{u} . \mathrm{v}$ shadowing of the in vitro transcription mixture of the stabilized RNA on $12 \%$ urea-denaturing PAGE. Single prominent band represents 2'-fluorine-modified RNA transcript

\section{SELEX}

SELEX is a process that generates aptamer, singlestranded DNA or RNA that binds to a myriad of target molecules such as proteins (Calik et al., 2009), amino acids (Geiger et al., 1996) and drugs (Jenison et al., 1994). SELEX consists of several steps, starting with the incubation of the random oligonucleotide library with the target, separation of the bound from unbound nucleic acids, followed by the amplification of the bound sequences.
Comprising of both RNA and DNA aptamer, RNA aptamer is favoured over the latter as the preparation of the RNA through in vitro transcription is much faster and less tedious compared to the conversion of the double stranded to singlestranded DNA. This RNA can be purified from in vitro transcription mixture by the use of spin column, which removes excess salt and enzymes. However, partial RNA transcripts can also be purified together with the full length transcripts, which can interfere with the binding of the correct-sized RNA fragment to the target protein in SELEX. 'Abortive' (N-1) or 'add-on' $(\mathrm{N}+1, \mathrm{~N}+2)$ RNA transcripts 
which are the partial fragments also produced amidst the correct full length of the transcript during in vitro transcription. Moreover, in some in vitro transcription reactions, prematurely terminated RNA transcripts are also generated as a result of the presence of sequences that resemble factor-independent transcription termination sequences. The utilization of phenol: chloroform in purifying RNA transcript is often associated with incomplete removal of phenol: chloroform, which can denature the target protein in SELEX cycle. Hence, we have employed fast method of purification to purify RNA transcript in one of the SELEX cycles that was carried out. In this strategy, the amplified PCR product subjected to in vitro transcription was purified subsequently from the urea-denaturing PAGE using the method. Having the size of 69 nucleotides (nt) long, this RNA fragment can elute passively from the polycarylamide gel upon heating at $50-60{ }^{\circ} \mathrm{C}$ in water. The RNA obtained from this purification is intact, as band of the expected size was obtained following reverse transcription and PCR amplification (Figure 3). The purified RNA is subjected to the next cycle of SELEX experiment, with the amount of the RNA reduced in each subsequent cycles of SELEX.

Another advantage of our method is the exclusion of the DNAse I treatment, as the RNA transcript and the corresponding PCR product migrate at different rates on the urea-denaturing PAGE. This enables selective and precise excision of the RNA fragment. As reported above, this purification protocol can also be utilized to obtain purified form of the RNA transcript tagged radioactively, in order to calculate the percentage of enrichment in each cycle of SELEX. Most of the aptamers produced from SELEX experiments are generated by 'trial and error' basis, as SELEX cycles have to be repeated several times if the putative aptamer acquired after certain cycles of SELEX binds target with very low binding affinity. Our RNA purification method can reduce the overall time of SELEX experiment (up to a day), more SELEX cycles can be carried out and repeated in case of 'failure' of SELEX. In one type of SELEX experiment, gel shift assay was performed after 4-5 cycles of SELEX. The band representing the proteinnucleic acid complex is crushed and the bound RNA can be obtained using the purification method reported. As this complex contains nucleic acid species that have already achieved reasonable binding affinity towards the target protein, these potential high affinity binders can be purified and enriched. These purified RNA can be reversetranscribed to produce first-strand cDNA, which is further amplified by PCR and subjected to in vitro transcription. As these 'potential' binders have been separated from other low affinity binders, another additional 3 to 4 cycle of SELEX will result in the emergence of eventual high affinity binding species. This strategy of in vitro selection can greatly increase the probability of 'fishing' the 'winner' sequences from the random oligonucleotide pool.

\section{Fig. 3}
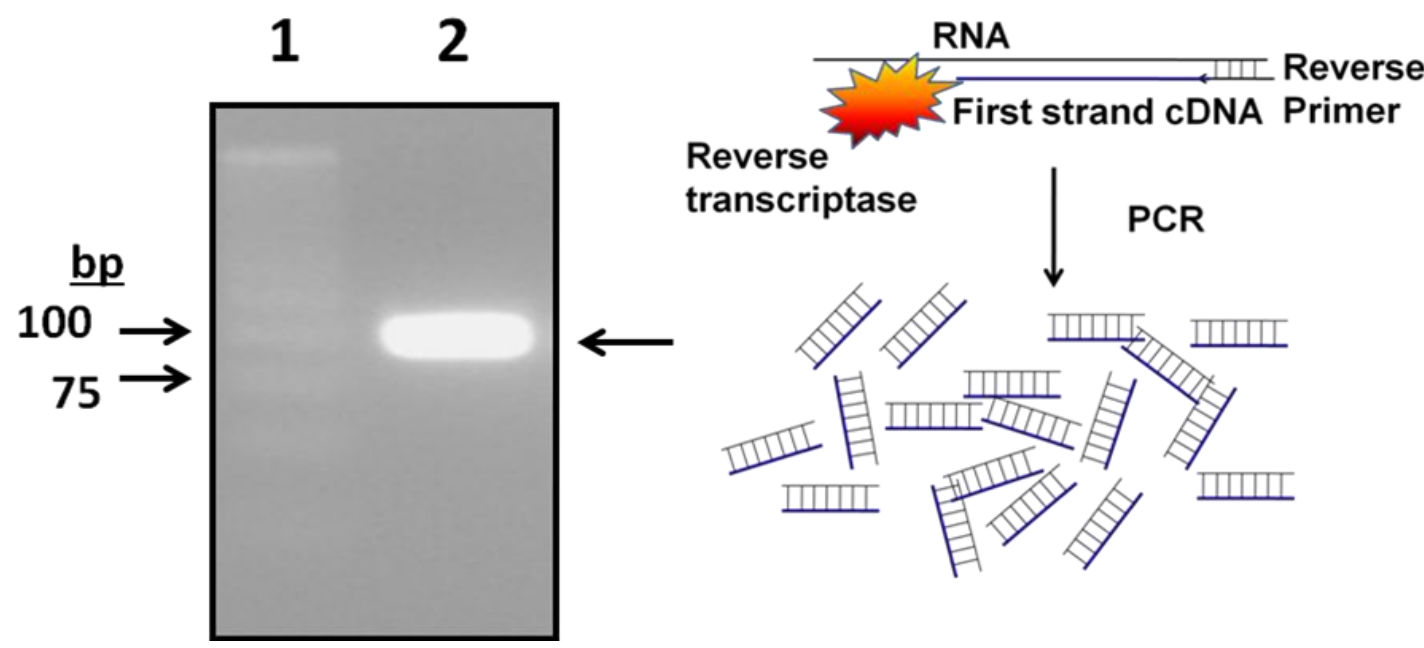

Figure 315 cycles of PCR amplification carried on the reverse-transcribed RNA from $2^{\text {nd }}$ SELEX cycle

Lane 1: 25 bp DNA ladder; Lane 2: 5 uL of the PCR product

\section{MAPPING OF RNA-PROTEIN INTERACTIONS}

The ability of the RNA molecules to form a wide variety of three dimensional structures such as G-quadruplex, stem loop and pseudoknot structures enables them to interact with protein. These interactions include protein-induced RNA folding, RNA-induced protein folding and co-induced folding. In the studies of RNA-protein interaction, it is important to identify the individual bases that interact with protein. Information gained on this will be useful for the design of further experimental methods to furnish information on the functional aspect of the RNA. Identification of the nucleotides that interact with the target protein can facilitate the trimming of the excess region of the RNA fragment that is not important for binding. This truncated RNA fragment is more feasible for applications such as biosensor development and nanotheranostics. 
In line assay is an assay that depends on the differential degradation of the RNA based on the structure. Phosphodiester bonds between bases that form the secondary structure are less susceptible to hydrolysis than those that are not involved in secondary structure formation, such as in stem loop structures. As the first step for this assay, the purified form of the RNA is radiolabeled, spontaneously cleaved and separated on the urea-denaturing PAGE. The pattern of spontaneous cleavage between the RNA incubated with and without target is compared. Another important assay of RNA-protein mapping studies is ethylnitrosourea (ENU) mapping. By means of alkylating process imparted by ENU, phosphate groups are ethylated to form ethyl phosphotriesters, which are very sensitive to partial alkaline hydrolysis reaction. As the phosphate groups of nucleotides that are important for binding to the proteins were modified, these molecules are lost and do not appear on the gel. As a result, those 'lost' nucleotides can be visualized as 'invisible' or 'faint' bands on the ureadenaturing PAGE. Another assay known as toe-printing or primer extension inhibition assay, is used for analysis of translational initiation complex. This assay enables identification of 3'-end region at one edge of the RNAprotein complex. In this assay, reverse transcription of the RNA of interest complexed with target protein is primed by complementary cDNA primer oligonucleotide with the aid of reverse transcriptase enzyme. As the reaction proceeds, cDNA synthesis is stopped at a point where collision occurs between the target protein and the enzyme reverse transcriptase. This results in single nucleotide resolution from the 3'-end region of the RNA-protein complex.

Equivalently, heel-printing analysis facilitates the identification of the other edge, which is the 5'-end region of the RNA-protein complex. In this assay, RNA-target protein complex is digested, in which fragment that is not bound to protein is removed. Following this, the RNA fragment bound to the protein is isolated and hybridized to single stranded antisense cDNA strand, followed by the addition of the 5'-labeled oligonucleotide primer and T4 DNA Polymerase. During the strand elongation, as the T4 DNA Polymerase unable to unwind the RNA-DNA hybrid, the polymerase stalls at the 5'-end region of the digested RNA fragment. This provides single nucleotide resolution of the 5 '-end region of the RNA-protein complex (Wolin and Walter, 1988). These various assays of RNA-protein interactions are highly dependent on the single nucleotide resolution of the properly folded RNA binding to protein with high affinity. Folding of RNA into compact 3-D structure aided by tertiary interactions between the secondary structures (helices, bulges and internal loop) requires the RNA to be intact. The presence of reaction enzymes, excess nucleotides or primers can distort the conformation of the folded RNA. Moreover, the presence of reaction enzymes that occupy the RNA fragments can serve as hindrance towards the binding of the target protein. In the case of toe-printing and heel-printing assay, the remaining trace amounts of labelling enzymes can prevent the binding of reverse transcriptase and $\mathrm{T} 7$ polymerase enzyme, respectively to the target RNA. Excess of ions such as $\mathrm{Mg}^{2+}$ and $\mathrm{Ca}^{2+}$, deriving from salt can impart charge repulsion with the amino acid residues of the target protein, obstructing the binding of RNA with the protein. These imply that a good small RNA purification method can go a long way for both RNA-target protein formation and good single nucleotide resolution on the urea-denaturing PAGE. As a proof-of-concept, we have carried out partial alkaline digestion as well as RNAse T1 digestion on the radiolabeled RNA purified using the protocol explained. As shown in Figure 4, the radiolabeled RNA purified using the method is seen as an intact band (Lane 1) on the $12 \%$ denaturing-urea PAGE. When partially treated with $0.1 \mathrm{M}$ of Tris-Cl, $\mathrm{pH} 9.0$, this partial alkaline digestion resolved the RNA into single nucleotides (Lane 2). Treatment with RNase T1 specifically cleaves phosphodiester bond next to the G nucleotide (Lane 3 ). Resolution of the RNA into single nucleotides indicates that the radiolabeled RNA purified by this method is intact and of good quality.

\section{Fig. 4}

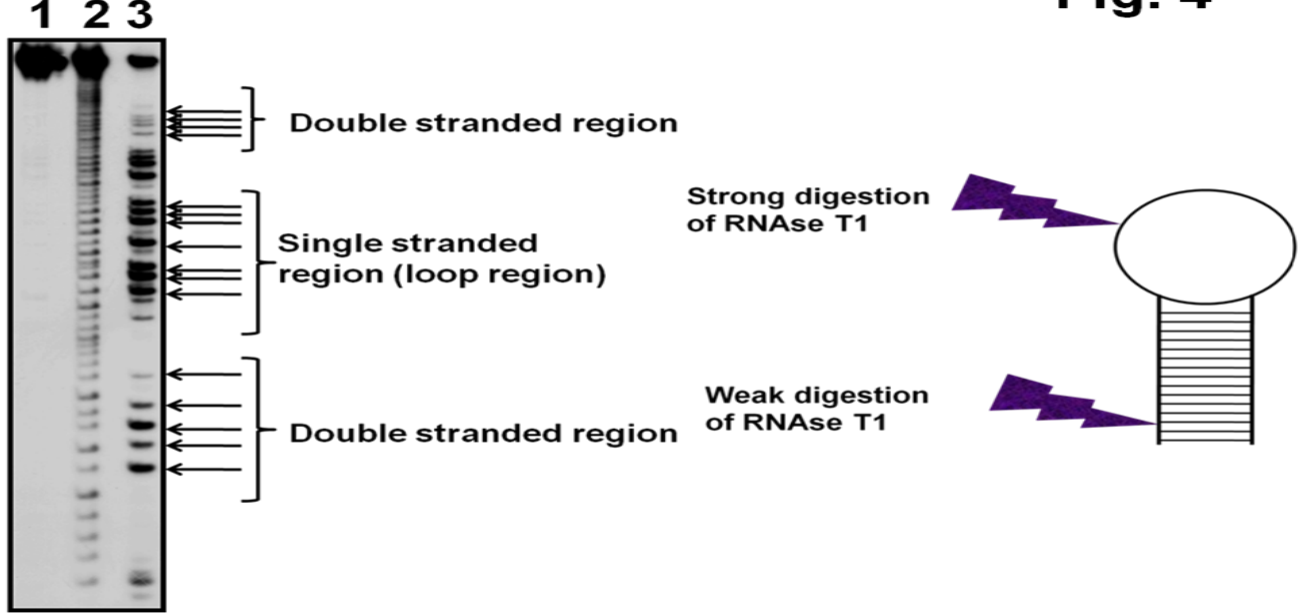

Figure 4 Partial alkaline and RNAse T1 digestion of the 114-mer RNA transcript

RNAse T1 cleaves specifically on the phosphodiester bond next to guanine $(\mathrm{G})$ nucleotide

The digestion is more prominent in the single stranded region (such as stem loop structure) compared to the double stranded region

Lane 1: RNA purified using the method. Lane 2: partial alkaline digestion. Lane 3: RNase T1 digestion 


\section{LABEL-FREE RNA-PROTEIN INTERACTIONS}

Surface Plasmon Resonance (SPR) is a phenomenon of excitation of surface plasmon (oscillation of electrons) by light or electromagnetic wavelength at the metal-dielectric interface. Absorption of any molecules on the surface of the metal-dielectric interface will change the reflectivity of the electromagnetic wavelength. Thus, the interaction between any biomolecules such as protein-protein (Shliom et al., 2000), RNA-protein (Katsamba et al., 2002) and DNAprotein interactions (Mischiati et al., 1999) can be monitored on the metal surface by monitoring the changes in reflectivity. Based on the SPR principle, Biacore technology which depends on the automated optical based detection system was created. This technology monitors the immobilization and interaction of biomolecules by sensing the interfacial refractive index change which occurs in close proximity to the metal surface. There are several Biacore chips available with different immobilization surfaces for biomolecules such as CM5 (carboxymethyl dextran surface), gold $(\mathrm{Au})$ and Streptavidin (SA) chips. In order to immobilize RNA on the surface of the chip, the RNA fragment has to be conjugated to functional groups that can form interactions with the functional groups on the surface of the chip. For example, conjugation of the RNA with biotin will enable successful immobilization of the RNA on the surface of streptavidin (SA) chip, owing to the high affinity of biotin-streptavidin complex formation $\left(1 \times 10^{-14}\right.$ $\mathrm{M})$. The RNA transcript is generated from the PCR product with one of the primers containing extended sequence (polyadenine tract) at one end. This sequence does not interfere with the structural conformation of the folded RNA, in fact ensures there is some space between the RNA and the surface of the platform to avoid steric hindrance. Next, the sequence tailored with biotin which is complementary to the extended sequence of the transcript is immobilized on the surface of the streptavidin chip. The subsequent addition of the RNA fragment will result in immobilization on the platform surface, as this extended sequence of the RNA forms duplex with the sequence linked to biotin. For efficient immobilization on the surface of the Biacore chip, the RNA fragment with additional bases at one end must be of high purity. Moreover, the presence of trace amounts of phenol or dithiothreitol can lead to changes in the dielectric environment on the surface of the chip, producing erroneous measurement of the refractive index change. The usual purification method that involves the usage of ethidium bromide in visualizing nucleic acid prior to purification can also exert false changes in the refractive index measurement. Thus our purification method which only uses water as the media for elution will result in RNA that is free from any excess salts or intercalating agents that can result in spurious measurement. Similar purification method can also be employed to prepare RNA transcript for waveguide mode sensors.

Functioning in a manner similar to SPR, excitation of the waveguide mode based sensor relies on the excitation of the waveguide mode, resulting in the changes of the reflectivity of the incidence light on the waveguide surface. Immobilization of RNA on the surface of the waveguide- mode sensor surface made up of $\mathrm{Au}$ (gold) and Silica $\left(\mathrm{SiO}_{2}\right)$ (Gopinath et al., 2010) can be achieved via thiolation. Similar to Surface Plasmon Resonance (SPR) application, our purification method can give rise to intact and pure RNA modified with functional groups for effective immobilization and subsequent capture of target protein. Being sensitive to the dielectric environment in the vicinity of the waveguide surface, any impurities can result in alteration of the dielectric environment, changing the reflectivity drastically. This results in the acquisition of false signal upon immobilization of a RNA fragment and its subsequent interaction with protein. As reiterated before, our purification method explained earlier removes impurities such as truncated nucleotides and enzymes. On the other hand, our fast method of small RNA purification can also be applied to preparing small RNA for monitoring RNAprotein interaction based on reflectivity measurement by UV-vis-NIR spectroscopy on the gold coated silica substrate. For conjugating RNA fragment to the surface of the sensor, similar method of immobilization carried out with Streptavidin biacore chip can be used, except that the functional group is thiol instead of biotin (Gopinath et al., 2010). UV-vis-NIR spectroscopy measurement is also as sensitive as SPR and waveguide, entailing the RNA to be pure from proteins and other oligonucleotides which can also give reflectivity.

\section{CONCLUSION}

The fast and cost-effective method of small RNA purification can be successfully used in various applications that involve RNA-protein interaction. As discussed earlier, it can be applied to both label and label-free applications of RNA-protein interaction analysis. In tandem with the burgeoning interest in developing RNA based biosensor, this method of RNA purification can also give rise to stabilized RNA incorporated with 2'-Fluoridine modified nucleotides, in a short period of time.

\section{ACKNOWLEDGEMENT}

This work was funded by USM RU Grant 1001/CIPPT/813043. We would like to thank Universiti Sains Malaysia for awarding Academic Staff Training Scheme to Citartan Marimuthu for this study.

\section{REFERENCES}

[1] Armstrong RN, Colyer HA, Mills KI (2012). Screening for miRNA Expression Changes Using Quantitative PCR (QPCR). Methods Mol Biol., 863: 293-302.

[2] Calik P, Balci O, Ozdamar TH (2009). Human growth hormone-specific aptamer identification using improved oligonucleotide ligand evolution method. Protein Expres. Purif., 69: 21-28.

[3] Chinni SV, Raabe CA, Zakaria R, Randau G, Hoe CH, Zemann A, Brosius J, Tang TH, Rozhdestvensky TS (2010). Experimental identification and characterization of 97 novel npcRNA candidates in Salmonella enterica serovar Typhi. Nucleic Acids Res., 17: 5893-5908.

[4] Citartan M, Tan SC, Tang TH (2012). A rapid and cost effective method in purifying small RNA. World JMicrobBiot., 28: 105-111. 
[5] Gambari R (1999). Interaction of the human NF-kappaB p52 transcription factor with DNA-PNA hybrids mimicking the NF-kappa B binding sites of the human immunodeficiency virus type 1 promoter, J. Biol. Chem., 274: 33114-33122.

[6] Gopinath SCB, Awazu K, Fujimaki M (2010). Detection of influenza viruses by a waveguide-mode sensor. Anal. Methods., 2: 1880-1884.

[7] Gopinath SCB, Balasundaresan D, Kumarevel T, Misono TS, Mizuno H, Kumar PKR (2008). Insights into anti-termination regulation of the hut operon in Bacillus subtilis: importance of the dual RNA-binding surfaces of HutP. Nucleic Acids Res., 36: 3463-3473.

[8] Gopinath SC, Kumaresan R, Awazu K, Fujimaki M, Mizuhata M, Tominaga J, Kumar PK (2010). Evaluation of nucleic acid duplex formation on gold over layers in biosensor fabricated using Czochralski-grown single-crystal silicon substrate. Anal Bioanal Chem., 398: 751-758.

[9] Gopinath SCB, Sakamaki Y, Kawasaki K, Kumar PKR (2006). An Efficient RNA Aptamer against Human Influenza B Virus Hemagglutinin. J. Biochem., 139: 837-846.

[10] Geiger A, Burgstaller P, von der Eltz H, Roeder A, Famulok M (1996). RNA aptamers that bind L-arginine with submicromolar dissociation constants and high enantioselectivity. Nucleic Acids Res., 24: 1029-1036.

[11] Jenison RD, Gill SC, Pardi A, Polisky B (1994). Highresolution molecular discrimination by RNA. Science., 263: $1425-1429$.

[12] Katsamba PS, Park S, Laird-Offringa IA (2002). Kinetic studies of RNA-protein interactions using surface plasmon resonance, Methods., 26: 95-104.

[13] Leontis NB, Lescoute A and Westhof E (2006). The building blocks and motifs of RNA architecture. Curr Opin Struct Biol., 16: 279-287.

[14] Mischiati C, Borgatti M, Bianchi N, Rutigliano C, Tomassetti M, Feriotto G, Gambari R (1999). Interaction of the human
NF-kappaB p52 transcription factor with DNA-PNA hybrids mimicking the NF-kappaB binding sites of the human immunodeficiency virus type 1 promoter. J. Biol. Chem., 274: 33114-33122.

[15] Ryder SP, Recht MI, Williamson JR (2008). Quantitative analysis of protein-RNA interactions by gel mobility shift. Methods Mol Biol., 488:99-115.

[16] Shliom O, Huang M, Sachais B, Kuo A, Weisel JW, Nagaswami C, Nassar T, Bdeir K, Hiss E, Gawlak S, Harris S, Mazar S, Higazi AA (2000). Novel interactions between urokinase and its receptor, J. Biol. Chem., 275: 24304-24312.

[17] Stockley PG (2009). Filter-binding assays. Methods Mol Biol., 543: $1-14$

[18] Tang TH, Rozhdestvensky TS, d'Orval BC, Bortolin ML, Huber H, Charpentier B, Branlant C, Bachellerie JP, Brosius J, Hüttenhofer A (2002). RNomics in Archaea reveals a further link between splicing of archaeal introns and rRNA processing. Nucleic Acids Res., 30: 921-30.

[19] Tang TH, Bachellerie JP, Rozhdestvensky T, Bortolin ML, Huber H, Drungowski M, Elge T, Brosius J, Hüttenhofer A (2002). Identification of 86 candidates for small nonmessenger RNAs from the archaeon Archaeoglobus fulgidus. Proc Natl Acad Sci U S A., 99: 7536-7541.

[20] Wolin SL and Walter P (1988). Ribosome pausing and stacking during translation of a eukaryotic mRNA. The EMBO Journal vol., 7: 3559 - 3569.

[21] Yamamoto R, Katahira M, Nishikawa S, Baba T, Taira K, Kumar PKR (2000). A novel RNA motif that binds efficiently and specifically to the Ttat protein of HIV and inhibits the trans-activation by Tat of transcription in vitro and in vivo. Genes Cells 5 371-388.

[22] Zuo G, Li W, Zhang J, Wang J, Wang W (2010). Folding of a Small RNA Hairpin Based on Simulation with Replica Exchange Molecular Dynamics. J. Phys. Chem. B., 114: $5835-5839$. 